Supporting Information

\title{
Pore size gradient effect in monolithic silica mesopore networks revealed by in-situ-SAXS-physisorption
}

\author{
Sebastian A. Kube ${ }^{\text {a,b }}$, Kevin Turke ${ }^{\text {a }}$, Rüdiger Ellinghaus ${ }^{\text {a }}$, Dirk Wallacher ${ }^{\text {c }}$, Matthias \\ Thommes $^{\mathrm{d}}$, and Bernd M. Smarsly ${ }^{\mathrm{a}, \mathrm{e}, *}$
}

${ }^{a}$ Institute of Physical Chemistry, Justus-Liebig-University Giessen, Heinrich-Buff-Ring 17, 35392 Giessen, Germany

${ }^{b}$ Department of Mechanical Engineering and Materials Science, Yale University, 15 Prospect Street, New Haven, CT 06511, United States

${ }^{\mathrm{c}}$ Helmholtz Center Berlin for Materials and Energy, Hahn-Meitner-Platz 1, 14109 Berlin, Germany

${ }^{\mathrm{d}}$ Institute of Separation Science and Technology, Department of Chemical and Bioengineering, University of Erlangen-Nuremberg, Egerlandstrasse 3, 91058 Erlangen, Germany

${ }^{\text {e }}$ Center for Materials Research (LaMa), Justus-Liebig-University Giessen, Heinrich-BuffRing 17, 35392 Giessen, Germany

*Corresponding Author, E-mail: Bernd.Smarsly@phys.chemie.uni-giessen.de 
Supporting Information: Pore size gradient effect in monolithic silica mesopore networks revealed by in-situ-SAXS-physisorption; Sebastian A. Kube, Bernd M. Smarsly, et al., Langmuir, 2020.
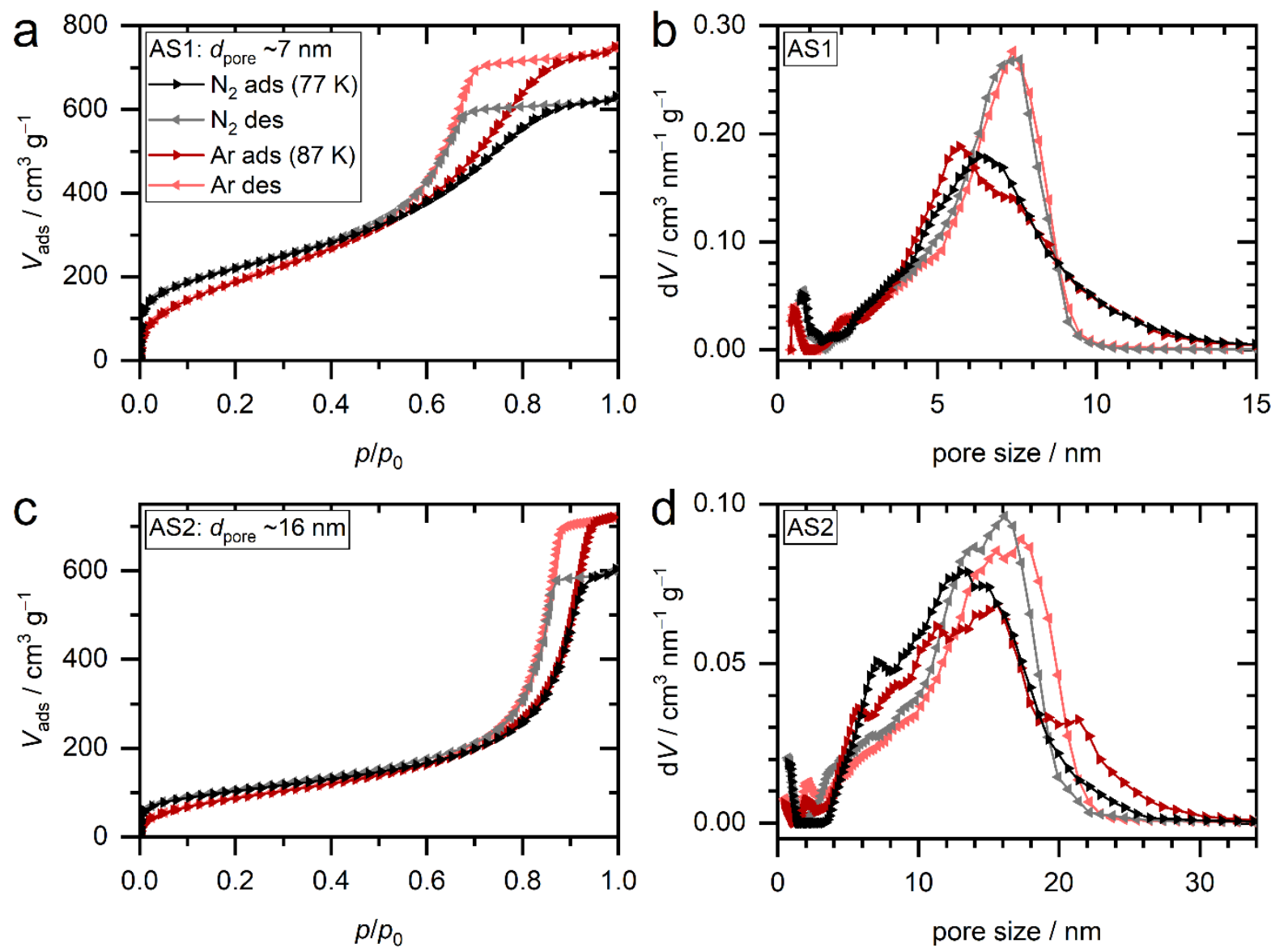

Figure S1. Preparatory test 2: $(\boldsymbol{a}, \boldsymbol{c})$ Nitrogen $(77 \mathrm{~K})$ and argon $(87 \mathrm{~K})$ physisorption isotherms of samples $A S 1$ and $A S 2$, respectively. $(\boldsymbol{b}, \boldsymbol{d})$ Corresponding pore size distributions as determined through NLDFT analysis. The good agreement of PSDs between adsorption and desorption, as well as the different adsorptives, suggests that pore blocking and cavitation do not appreciably contribute to pore emptying in AS1, AS2, and the main sample, which size-wise falls in between. 
Supporting Information: Pore size gradient effect in monolithic silica mesopore networks revealed by in-situ-SAXS-physisorption; Sebastian A. Kube, Bernd M. Smarsly, et al., Langmuir, 2020.

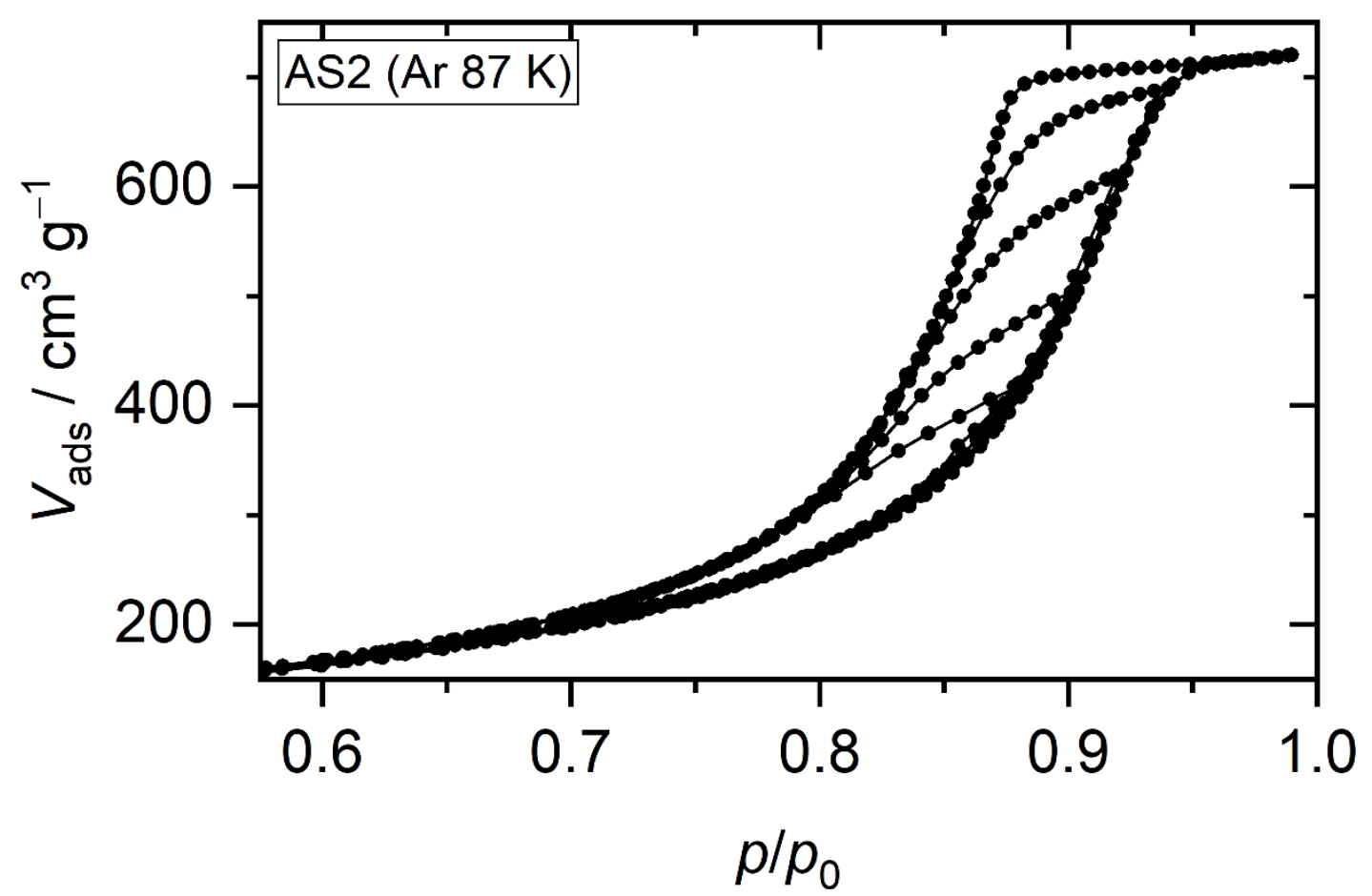

Figure S2. Argon physisorption isotherm measured at $87 \mathrm{~K}$ for AS2 including three scanning desorption isotherms. Similar to the Krypton isotherm measured for the main sample and presented in Figures $5 a$ and $b$, the scanning desorption isotherms do not converge in the lower hysteresis closing point, but rather merge with the main desorption isotherm before. The merging point shifts to higher relative pressures the greater the onset pressure of the respective scanning isotherm. We conclude that this behavior, which we interpret and explain through the presence of a mean pore size gradient as illustrated in Figure 8, is generally found in this monolithic silica material under study, regardless of the range covered by the pore size distribution (mean pore size: $\sim 13 \mathrm{~nm}$ in main sample, $16 \mathrm{~nm}$ in AS2) and the adsorptive gas used (Kr in main sample, Ar in AS2 here in Fig. S2). 
Supporting Information: Pore size gradient effect in monolithic silica mesopore networks revealed by in-situ-SAXS-physisorption; Sebastian A. Kube, Bernd M. Smarsly, et al., Langmuir, 2020.

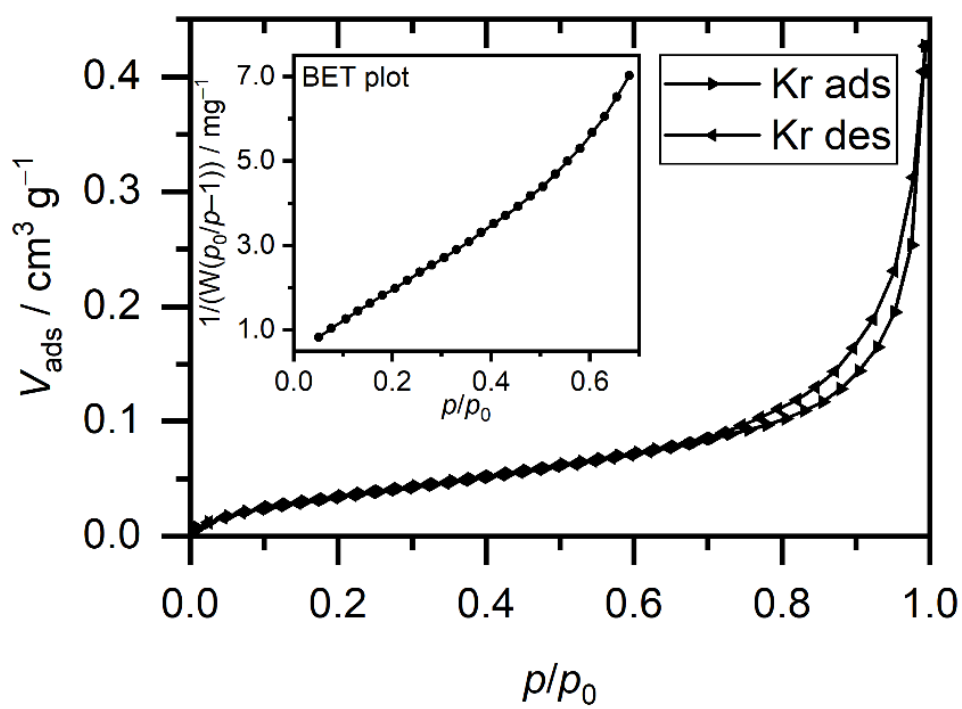

Figure S3. Preparatory test 3: Kr isotherm measured on macroporous silica absorbent at $120 \mathrm{~K}$. 
Supporting Information: Pore size gradient effect in monolithic silica mesopore networks revealed by in-situ-SAXS-physisorption; Sebastian A. Kube, Bernd M. Smarsly, et al., Langmuir, 2020.
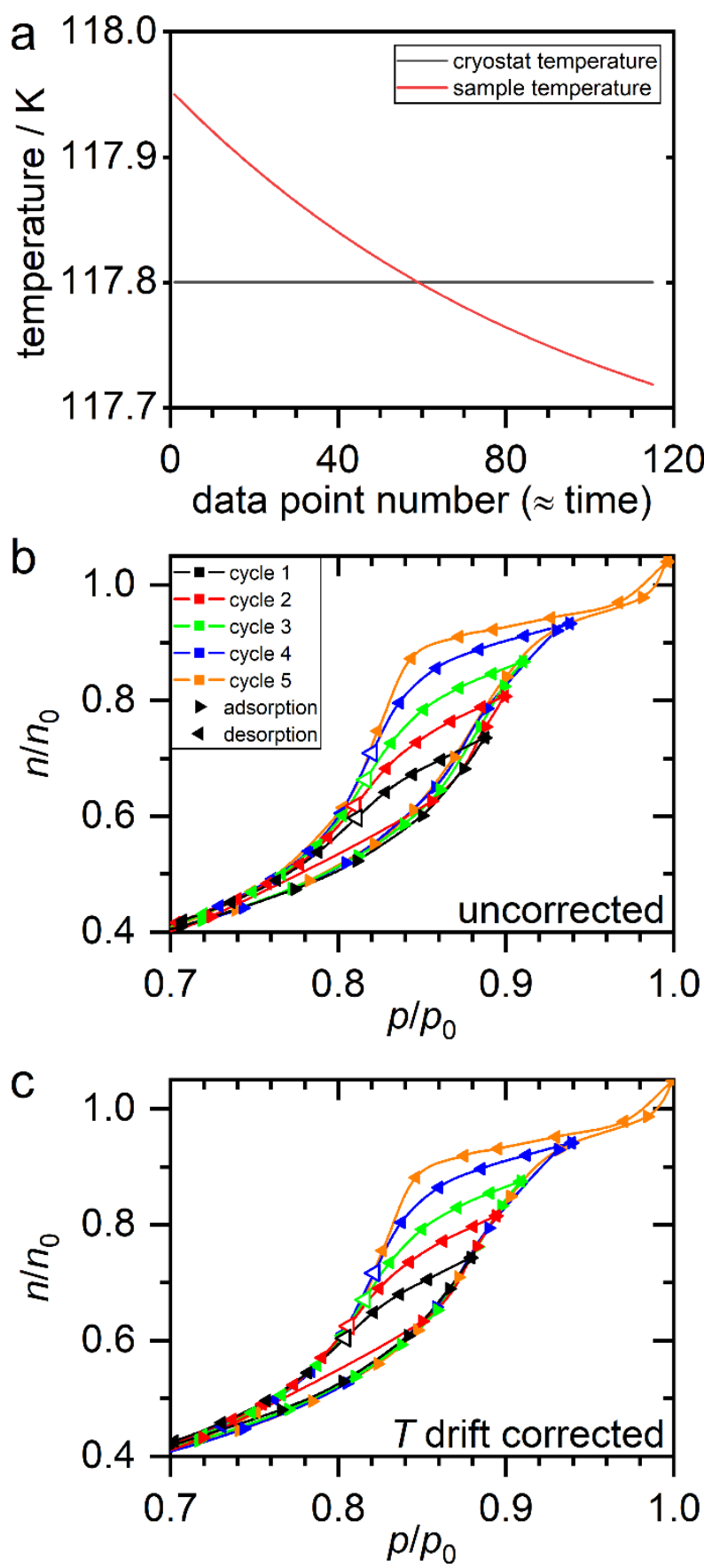

Figure S4. Correction of the in-situ adsorption isotherm by temperature drift: (a) The temperature of the cryostat thermometer (black line) as measured at the bottom of the sample cell, and the sample temperature (red line) as corrected from the pressure drifts at equilibrium conditions over the time of the experiment. To correct for the slightly changing sample temperature over the time of the in-situ investigation, the measured equilibrium pressures of all isotherm data points were related to individual reference pressures $P_{0}(T)$ according to the saturation vapor pressure curve of krypton as calculated from the Antoine equation ${ }^{1}$ with the parameters $A=4.2064, B=539.004, C=8.855$. (b, c) Kr in-situ adsorption isotherm before and after correction for the temperature drift, respectively. Proper coincidence of the adsorption branches is achieved. 
Supporting Information: Pore size gradient effect in monolithic silica mesopore networks revealed by in-situ-SAXS-physisorption; Sebastian A. Kube, Bernd M. Smarsly, et al., Langmuir, 2020.

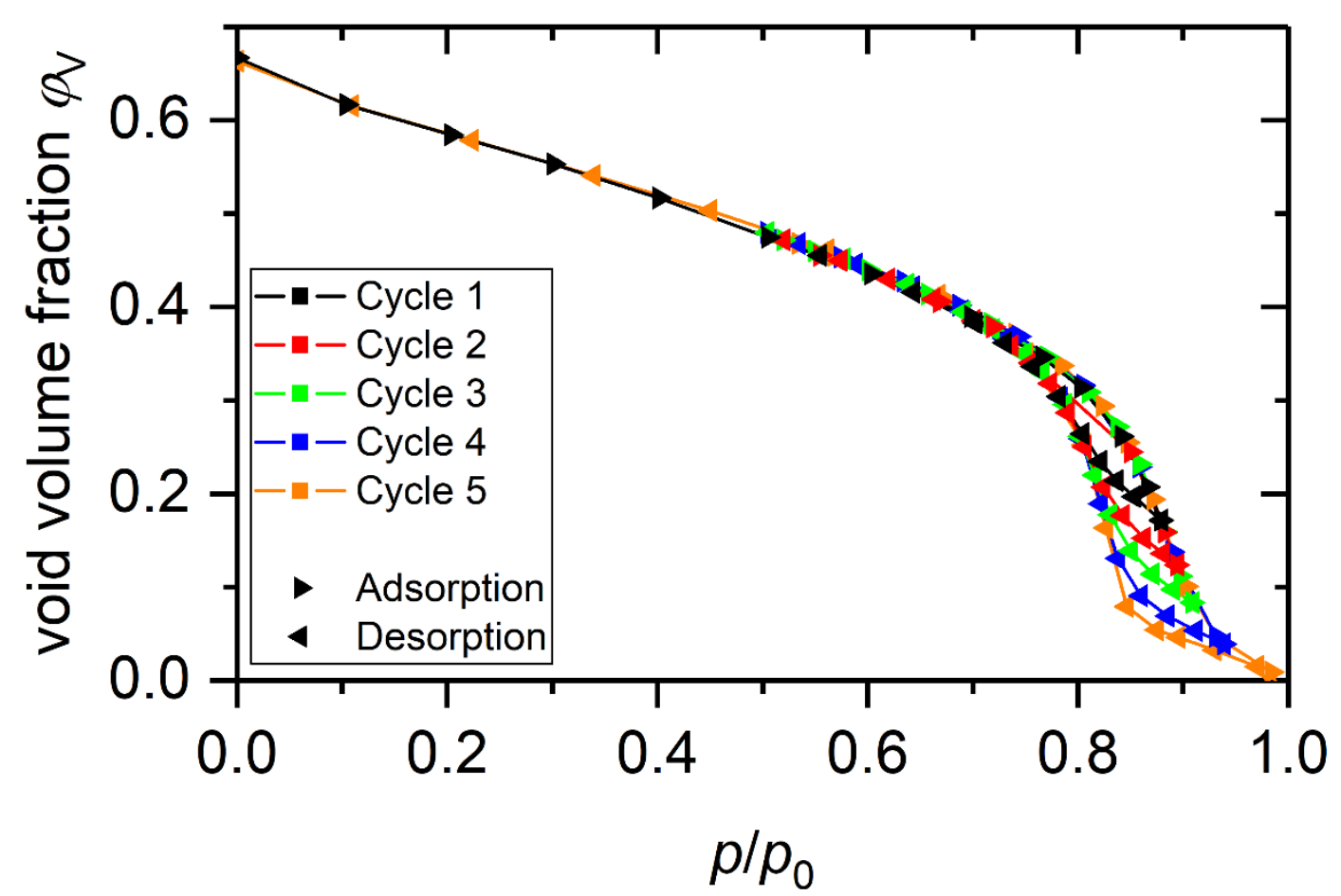

Figure S5. Change of the void volume fraction $\varphi_{\mathrm{V}}$ with relative pressure as calculated by equation (5) from the in-situ Kr isotherm. 
Supporting Information: Pore size gradient effect in monolithic silica mesopore networks revealed by in-situ-SAXS-physisorption; Sebastian A. Kube, Bernd M. Smarsly, et al., Langmuir, 2020.
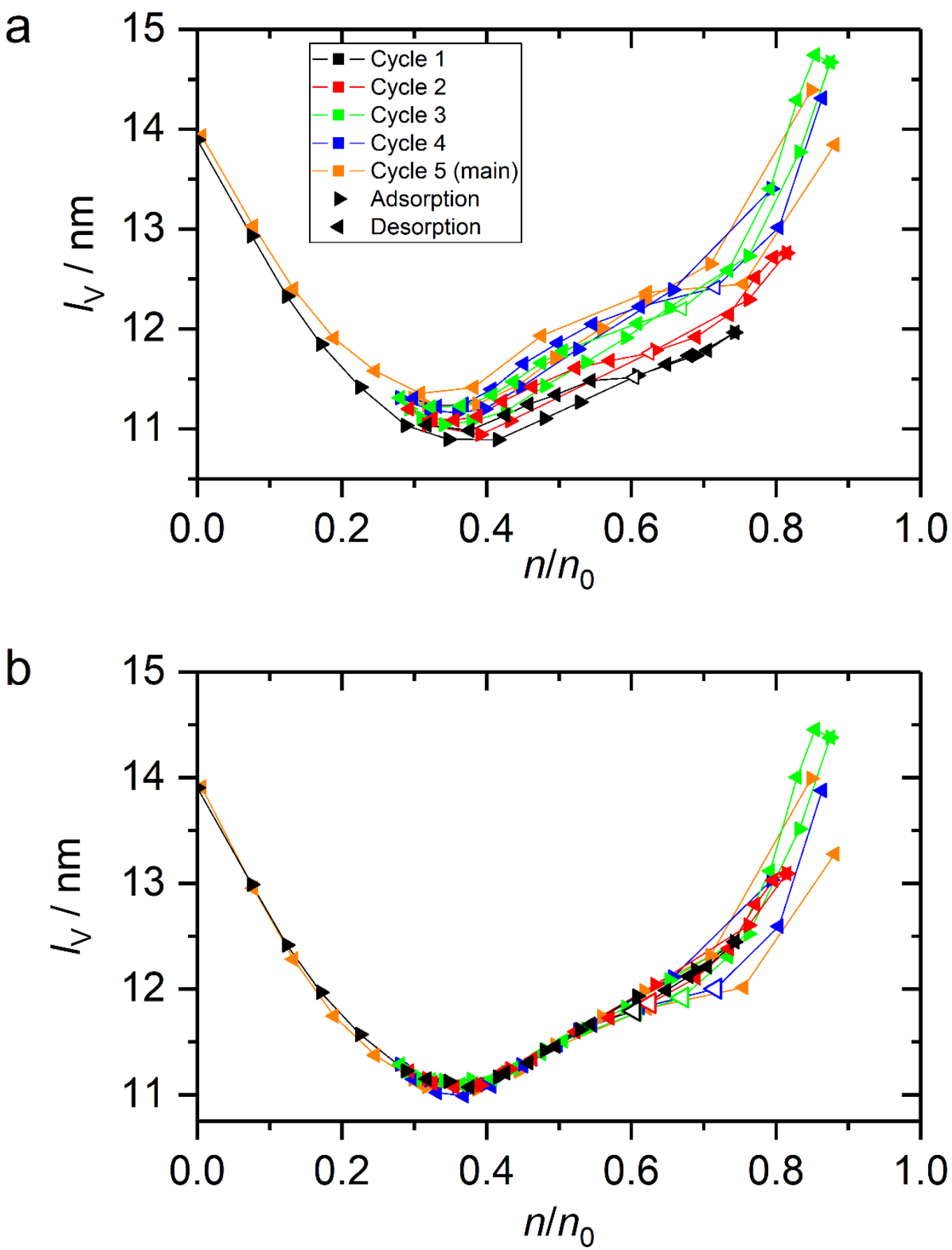

Figure S6. Correction of the $\mathbf{l}_{\mathbf{v}-n / n_{0}}$ isotherm: (a) Uncorrected isotherms. (b) Corrected isotherms: The adsorption branches and the desorption branch segments below the lower hysteresis closing point must coincide, since according to the physisorption isotherm (Fig. 5a and b) identical filling states are attained here. These segments were therefore linearly shifted into middle coincidence. The desorption branch segments above the lower hysteresis closing point correspond to differing filling states and must therefore not be forced into coincidence. They must, however, be shifted so that their start and endpoints properly connect with the corrected preceding and following segments. The resulting $l_{V-n} / n_{0}$ isotherm then displays the qualitatively correct behavior that agrees with the temperature corrected physisorption isotherm. The associated quantitative error is minimal. 

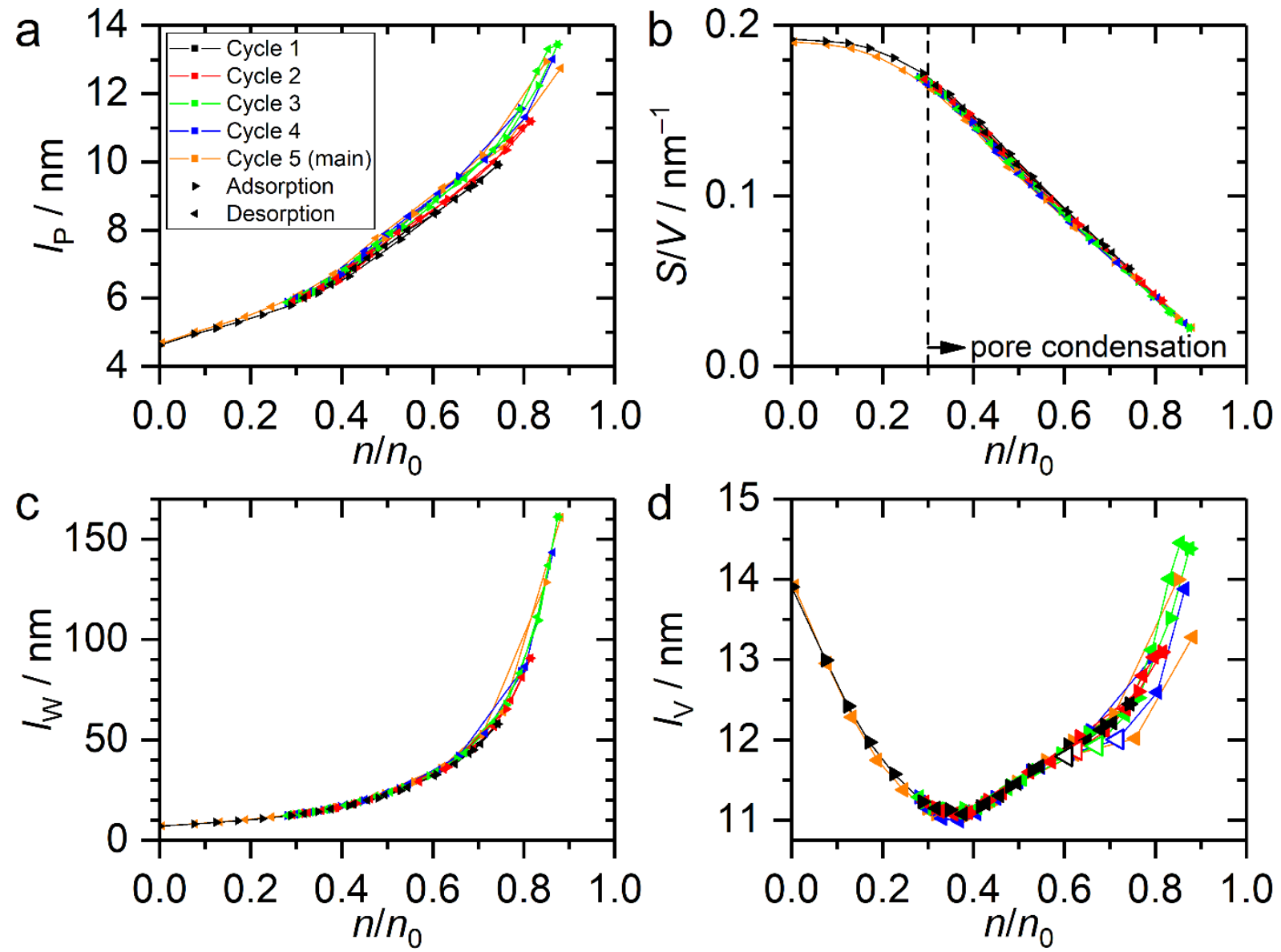

Figure S7. Characteristic CLD descriptors plotted against amount adsorbed $n / n_{0}:$ (a) Porod length $l_{\mathrm{P}}$. (b) Mesopore surface to volume ratio $S / V$. (c) Mean wall chord length $l_{\mathrm{W} .}$ (d) Mean void chord length $l_{\mathrm{V}}$, isotherms corrected for coincidence. 
Supporting Information: Pore size gradient effect in monolithic silica mesopore networks revealed by in-situ-SAXS-physisorption; Sebastian A. Kube, Bernd M. Smarsly, et al., Langmuir, 2020.

\section{S8: A detailed discussion complementing section 3.8: Understanding the $l_{v-n} / n_{0}$ plot (Fig. 8b)}

We use the illustrations in Figure 8c to explain the observed behavior schematically represented in Figure $8 \mathrm{a}$ and $8 \mathrm{~b}$. Beginning at point (A), both $p / p_{0}=1$ and $n / n_{0}=1$. According to eq. $4, l_{\mathrm{V}}$ diverges to infinity here, since $\varphi_{\mathrm{V}}=0$ and $l_{\mathrm{P}}$ diverges to infinity. Because the entire pore network is filled, only those mesopores which sit directly at the external surface (blue pores) are connected to the free gas phase via a direct path.

Then, as we move along the path (A)-(B1)-(C)-(D) of the main desorption isotherm, the pore network progressively empties. However, only such pores that are connected to the external free gas phase through a series of other empty pores can empty. These pores are again depicted in blue and empty via the external evaporation mechanism. Their liquid-gas interface constitutes the meniscus that progressively recedes into the pore network and at the same time blocks the metastable condensate in larger pores found more inwards from emptying (larger black pores). This blocking builds up the imbalance between larger blocked metastable pores and smaller empty pores, by which $l_{\mathrm{v}}$ assumes lower values on the main desorption curve than on the adsorption curve. At point (D), all mesopores contributing to hysteresis in the network have emptied (not depicted) and accordingly the lower closing point of hysteresis has been reached.

Next, instead of beginning at (A), we begin at (B2), the onset of the scanning desorption isotherm. Up to (B2) pores were independently filled with condensate depending on their size. At (B2) we therefore find that the network filling state can be divided into three regions as illustrated: At the center of the network (left), all pores are filled due to their small size. They constitute the densely filled core of the network. More outward (middle), some of the pores are filled whereas others are not, since in this region the mean pore size is close to the threshold of condensation, but pore sizes are still distributed randomly. These pores constitute the semi-dense shell of the network. Finally, towards the external surface pores are empty, since they are too large for adsorbate to condense at the given pressure.

(B2) is found at the same relative pressure as (B1), since both configurations have the same external meniscus position, i.e. the same set of pores with a direct path to the external gas phase (blue pores). 
Supporting Information: Pore size gradient effect in monolithic silica mesopore networks revealed by in-situ-SAXS-physisorption; Sebastian A. Kube, Bernd M. Smarsly, et al., Langmuir, 2020.

The amount adsorbed, however, is lower for (B2) than for (B1) because of the various empty pores in the semi-dense region. Owing to the presence of empty pores in the semi-dense shell, a second pore type with different connection characteristics exists (purple pores): Unlike the blue pores, these pores are not connected to the external gas phase. Instead, they are connected to the internal gas phase of neighboring empty pores. Accordingly, these pores cannot empty via the external evaporation mechanism, but instead via the internal evaporation mechanism.

Comparing (B1) to (B2), we find that there is a significantly larger number of pores with an available evaporation path in (B2), thanks to the added possibility of internal evaporation. Desorption must therefore occur faster in (B2), which is why the slope $\mathrm{d}\left(n / n_{0}\right) / \mathrm{d}\left(p / p_{0}\right)$ is higher in point (B2) of the physisorption isotherm than in (B1). Also, since the purple pores lie closer to the network center and are accordingly smaller than the blue pores, the average size of all pores which can empty (blue + purple) is smaller for (B2) than for (B1). In consequence, the reduction of the mean void pore size and $l_{\mathrm{V}}$ per amount desorbed is greater when desorbing from (B2) than from (B1) and therefore the slope of the $l_{\mathrm{V}}$ curve is steeper when desorbing from (B2). Finally, as already observed in the previous section, the slope of the $l_{\mathrm{V}}$ segment from (B2) to (C) is steeper than the slope of the adsorption curve in the same range. This is of course due to pore blocking, i.e. blue pores blocking larger black pores towards the center from emptying, by which the average void pore becomes smaller than on the adsorption branch for the same $n / n_{0}$.

In the next step we want to understand why the scanning isotherm merges with the main isotherm before reaching the lower closing point, that is before all mesopores contributing to hysteresis have emptied. How can the system reach an identical state at (C), coming from (B1) or (B2) regardless? From (B1) to (C) the meniscus of blue pores continuously recedes and evaporation occurs exclusively through external evaporation. By contrast, at (B2) a semi-dense shell exists, within which additional internal evaporation occurs into internal empty pores. Moving from (B2) to (C) the semi-dense shell diminishes, because no new internal empty pores can be formed in the dense core; as the meniscus of blue pores recedes into the network, internal empty pores and the neighboring purple pores are entirely depleted until point $(\mathrm{C})$ is reached. Here, only a dense network core blocked by the blue meniscus pores remains, 
Supporting Information: Pore size gradient effect in monolithic silica mesopore networks revealed by in-situ-SAXS-physisorption; Sebastian A. Kube, Bernd M. Smarsly, et al., Langmuir, 2020.

which is a situation found identically along the main desorption isotherm. From (C) to (D) to (E) the meniscus then recedes further into the network until hysteresis is closed and all mesopores have emptied.

Matching the above explanation, we observe that generally the merging point (C) shifts to higher or lower values of $n / n_{0}$ the higher or lower $n / n_{0}$ is at the onset of the scanning isotherm, respectively. In Figure 7 , for example, the onset of scanning isotherm 3 lies at lower $n / n_{0}$ than the onset of scanning isotherm 4 . Accordingly, the merging point of isotherm 3 also lies at lower $n / n_{0}$ than the merging point of isotherm 4. The same is observed for sample AS2 under argon physisorption in Figure S2. Within our pore network model, this means that for a lower $n / n_{0}$ at the scanning onset the meniscus lies more inward than illustrated in (B2). Consequently, upon desorption the meniscus must then recede further inward than shown in $(\mathrm{C})$, to fully deplete the semi-dense shell.

Finally, we note again that both in the physisorption isotherm (Fig. 5b) and in the plot of $l_{\mathrm{v}-} p / p_{0}$ (Fig. 6d) the rate of desorption and change of $l_{\mathrm{V}}$ with pressure is reduced upon passing the merging point, as indicated by the coinciding inflexion point and kink, respectively. This again is a consequence of the fact that the number of pores which can empty through evaporation is significantly higher from (B2) to (C) than beyond (C), so that network emptying is slowed once (C) is passed.

\section{$\underline{\text { References }}$}

1. Antoine, C., Vapor Pressure: a new relationship between pressure and temperature. Comptes Rendus des Séances de l'Académie des Sciences (in French) 1888. 